\title{
Erratum to: Chronic exposure to agmatine results in the selection of agmatine-resistant hepatoma cells
}

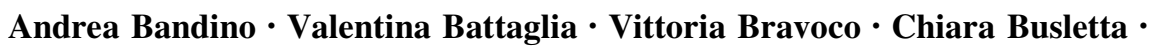 \\ Alessandra Compagnone $\cdot$ Carlo Cravanzola $\cdot$ Floriana Meli $\cdot$ Enzo Agostinelli $\cdot$ \\ Maurizio Parola $\cdot$ Sebastiano Colombatto
}

Published online: 22 February 2012

(C) Springer-Verlag 2012

Erratum to: Amino Acids (2012) 42:769-774

DOI 10.1007/s00726-011-0993-0

In the original publication of this article, the first and last names of authors were incorrectly published. The correct author names are given below:

Andrea Bandino - Valentina Battaglia · Vittoria Bravoco · Chiara Busletta $\cdot$ Alessandra Compagnone

Carlo Cravanzola · Floriana Meli - Enzo Agostinelli · Maurizio Parola $\cdot$ Sebastiano Colombatto

The correct affiliation of Enzo Agostinelli is:

Istituto Pasteur-Fondazione Cenci Bolognetti, Department of Biochemical Sciences, SAPIENZA University of Rome and CNR, Institute Biology and Molecular Pathology, Piazzale Aldo Moro 5, 00185, Rome, Italy

The online version of the original article can be found under doi:10.1007/s00726-011-0993-0.

\footnotetext{
A. Bandino · V. Bravoco - C. Busletta - A. Compagnone .

C. Cravanzola $\cdot$ F. Meli $\cdot$ M. Parola $\cdot$ S. Colombatto $(\square)$

Department of Experimental Medicine and Oncology,

University of Torino, via Michelangelo 27B, 10126 Turin, Italy

e-mail: sebastiano.colombatto@unito.it

V. Battaglia

Department of Biological Chemistry, University of Padua,

Viale G. Colombo 3, 35121 Padua, Italy

E. Agostinelli

Istituto Pasteur-Fondazione Cenci Bolognetti,

Department of Biochemical Sciences, SAPIENZA University

of Rome and CNR, Institute Biology and Molecular Pathology,

Piazzale Aldo Moro 5, 00185 Rome, Italy
} 